\title{
PHOSPHATATION DE RÉSIDUS MINÉRAUX
}

Blandine Bournonville *, Ange Nzihou *, Patrick Sharrock **, Guy Depelsenaire ***

* Laboratoire de génie des procédés des solides divisés, UMR CNRS 2392, EMAC

** IUT de chimie de Castres - Université Paul Sabatier

*** Solvay - HSE - DCRT Belgique

Solvay a breveté un nouveau procédé de stabilisation de résidus minéraux contaminés par des métaux lourds et des composés organiques dont l'une des étapes fondamentales est le traitement chimique à l'acide phosphorique. Son objectif est de former des phosphates de calcium de grande stabilité chimique et thermique, capables d'incorporer dans leur structure cristalline les métaux lourds. L'étude de la cinétique de phosphatation montre que la réaction est exothermique. Les phosphates calciques formés enrobent les particules les plus inertes. L'énergie d'activation faible $(20 \mathrm{~kJ} / \mathrm{mol})$, caractéristique d'une réaction acide-base, est proche de celle de la réaction avec le carbonate de calcium. Les expérimentations menées et les paramètres observés indiquent que la phosphatation peut être généralisée pour tous les résidus minéraux et constituer une première étape dans l'insolubilisation effective des métaux lourds. Ceci permet d'envisager pour le futur une possible revalorisation des résidus minéraux phosphatés et calcinés comme matière première secondaire.

The incineration of municipal solid waste generates mineral residues which contain soluble chlorides and heavy metal pollutants. The mineral residue is currently landfilled after a cement solidification. An alternative treatment, the new patented by Solvay process uses phosphoric acid to stabilise mineral residue by the formation of stable minerals such as calcium phosphates which are insoluble in natural environments and can incorporate heavy metals inside their crystalline structure. Two different water-washed municipal solid waste incinerator mineral residues are investigated during the phosphate treatment. Phosphoric acid reacts exothermically with mineral residues with rapid kinetics of the dissolution-precipitation type. Second order rate is observed with respect to the phosphate concentration and activation energies for the phosphate reaction are found to be small (near $20 \mathrm{~kJ} / \mathrm{mol}$ ). Mineral residues react with phosphoric acid mainly as calcium carbonate does. Precipitated amorphous calcium phosphates coat the more inert particles (silicoaluminates, silicates, and calcium sulphates...). The two mineral residues with different compositions show similar behaviour. This indicates the phosphate stabilisation procedure can be widely applied. This chemical treatment can be considered as a first essential step in the effective insolubilisation of extractable trace heavy metals in a mineral material that could find in the future an beneficial reuse after further processing as secondary raw material.

\section{INTRODUCTION}

Les fumées acides issues de l'incinération des ordures ménagères et des déchets industriels sont chargées en fines particules. Elles sont dépoussiérées par des électrofiltres ou des filtres à manches qui retiennent les cendres volantes $(\mathrm{CV})$ contenant des quantités non négligeables de métaux lourds et de chlorures. Ces CV doivent être stabilisées pour permettre leur stockage en centre d'enfouissement technique (CET) de classe I. Traditionnellement des liants hydrauliques sont utilisés pour la stabilisation des cendres volantes. Cependant, certains problèmes de stabilité au cours du temps se posent, notamment à cause de la présence des chlorures et sulfates qui à court et moyen terme, par départ (chlorures) ou néoformations minérales induites (aluminosulfates de calcium), fragilisent les matrices minérales formées [I].

Un traitement alternatif de stabilisation des cendres volantes par phosphatation est proposé. Après élimination des sels solubles qui sont recyclés dans une filière de Solvay, le procédé consiste en un inertage physicochimique ${ }^{[2]}$. Ce procédé est fondé sur le principe de l'incorporation des polluants dans une matrice phosphatée stable. En effet, les phosphates, notamment les phosphates calciques comme l'apatite $\left[\mathrm{Ca}_{10}\left(\mathrm{PO}_{4}\right)_{6}(\mathrm{OH})_{2}\right]$, sont des composés qui ont, par les nombreuses solutions solides possibles dans ce groupe minéral, une grande capacité de piégeage des métaux toxiques. En outre 
comme ils sont, dans les conditions physico-chimiques du milieu naturel, peu solubles ${ }^{[3,4]}$, ils constituent potentiellement des pièges durables. Quatre étapes composent le procédé de stabilisation des cendres volantes. Les sels solubles des cendres (chlorures, sulfates alcalins) sont récupérés dans des conditions de $\mathrm{pH}$ bien définies par un lavage suivi d'une filtration. De l'acide phosphorique est alors ajouté aux cendres volantes ainsi débarrassées de leur fraction soluble. L'ensemble est ensuite calciné pour éliminer les dioxines/furanes, assurer une bonne cristallisation des phosphates et ainsi améliorer leur stabilité. L'inertage ainsi proposé vise à diminuer le potentiel polluant des résidus de procédés thermiques, en vue de les stocker dans des conditions moins contraignantes que celles du CET de classe I ou, dans une hypothèse optimale, de les valoriser comme matières premières secondaires ${ }^{[2]}$.

L'étape fondamentale du procédé repose sur un traitement chimique : la phosphatation des cendres volantes. La compréhension de ce traitement est nécessaire pour optimiser les conditions opératoires. Mais, les cendres volantes apparaissant comme des produits hétérogènes constitués majoritairement d'aluminosilicates, d'oxydes, de sulfates, de carbonates, les processus de phosphatation par l'acide phosphorique s'avèrent complexes. Pour mieux les comprendre et mieux les expliquer, des réactions de phosphatation ont également été réalisées avec différents composés purs de référence présents dans les cendres volantes. Parmi les composés étudiés, le carbonate de calcium a une importance particulière compte tenu de son omniprésence dans les cendres de ce type et de sa disponibilité pour la formation de phosphates de type apatitique.

\section{MATÉRIEL ET MÉTHODES}

\section{Caractérisation physico-chimique des cendres volantes}

Les deux CV étudiées (CVS et CVO) proviennent de deux usines d'incinération d'ordures ménagères (UIOM) et sont issues d'un procédé de dépoussiérage par électro-filtre (première filtration) localisé en amont du système de traitement des fumées. Elles ont été choisies parmi des échantillons sélectionnés en Europe en raison de leur représentativité.

Avant tout essai de phosphatation, et conformément au procédé, la fraction soluble des $\mathrm{CV}$ a été préalablement extraite dans des conditions opératoires contrôlées ( $\mathrm{pH}$, rapport solide/liquide).

La composition chimique des deux cendres a été obtenue par fluorescence $X$ et méthode chimique pour les éléments majeurs et par torche à plasma pour les éléments mineurs. Le tableau I récapitule les données et montre que les CVS présentent une quantité importante de sulfate et environ deux fois plus de métaux lourds

\begin{tabular}{|c|c|c|c|c|c|}
\hline & $\mathrm{Al}_{2} \mathrm{O}_{3}$ & $\mathrm{CaO}$ & $\mathrm{Fe}_{2} \mathrm{O}_{3}$ & $\mathrm{SiO}_{2}$ & $\mathrm{SO}_{4}$ \\
\hline CVS & $9 \%$ & $25 \%$ & $8 \%$ & $18 \%$ & $20 \%$ \\
\hline CVO & $17 \%$ & $25 \%$ & $2 \%$ & $28 \%$ & $2 \%$ \\
\hline & $\mathrm{Cr}$ & $\mathrm{Cu}$ & $\mathbf{P b}$ & & $\mathrm{Zn}$ \\
\hline CVS & I 126 ppm & $2552 \mathrm{ppm}$ & $12500 \mathrm{pp}$ & & 28500 ppm \\
\hline CVO & 513 ppm & I 022 ppm & $3731 \mathrm{pp}$ & & 12417 ppm \\
\hline
\end{tabular}

que les CVO. Dans les deux cas, les éléments majeurs sont le calcium, le silicium et l'aluminium.

Les phases minérales majeures déterminées par diffraction des rayons $\mathrm{X}$ sont l'anhydrite $\left[\mathrm{CaSO}_{4}\right]$, la gehlenite $\left[\mathrm{Ca}_{2} \mathrm{Al}_{2} \mathrm{SiO}_{7}\right]$ et le carbonate de calcium $\left[\mathrm{CaCO}_{3}\right]$. Du quartz $\left[\mathrm{SiO}_{2}\right]$, des plagioclases $\left[(\mathrm{Ca}, \mathrm{Na})(\mathrm{Si}, \mathrm{Al})_{4} \mathrm{O}_{8}\right]$ et des phosphates peu cristallins sont également présents. Dans l'échantillon lavé, à l'exception des chlorures éliminés, les phases minéralogiques majeures déterminées correspondent à celles généralement répertoriées dans les cendres volantes non lavées [5].

La distribution granulométrique des cendres volantes déterminée par un granulomètre laser Mastersizer 2000 de Malvern montre un diamètre moyen des particules de $24 \mu \mathrm{m}$ pour les CVS et de $44 \mu \mathrm{m}$ pour les CVO, ce qui correspond certainement à des agrégats. Les masses volumiques analysées avec un pycnomètre Accupyc 1330 de Micromeritics sont de $2717 \mathrm{~kg} / \mathrm{m}^{3}$ pour les CVS et de $2489 \mathrm{~kg} / \mathrm{m}^{3}$ pour les CVO. La surface spécifique $\mathrm{Sp}$ des cendres volantes déterminée par la méthode BET sur un appareil Gemini de Micromeritics est de $10,7 \mathrm{~m}^{2} / \mathrm{g}$ pour les CVS et I5,7 m²/g pour les CVO.

\section{Protocole expérimental}

La phosphatation des cendres volantes et du $\mathrm{CaCO}_{3}$ a été étudiée dans un réacteur fermé de I litre à température contrôlée. Le contrôle des paramètres essentiels pour l'essai $(\mathrm{pH}$, température, masse d'acide phosphorique ajouté, vitesse d'agitation...) et l'acquisition des données sont effectuées au système automatisé Labmax, de Mettler Tolédo qui asservit le réacteur expérimental. Le mélange réactionnel est composé de 37l,4 g d'eau déminéralisée, de $200 \mathrm{~g}$ de cendres volantes (CVS ou $\mathrm{CVO}$ ) ou de $90 \mathrm{~g}$ de $\mathrm{CaCO}_{3}$ (99\% minimum de Fluka et Aldrich) et de 16 à $32 \mathrm{~g}$ d'acide phosphorique $\left(\mathrm{H}_{3} \mathrm{PO}_{4}\right.$ à $85 \%$ minimum Normapur de Prolabo). La température de la réaction est variable de 20 à $80^{\circ} \mathrm{C}$ et la suspension est agitée par un ruban hélicoïdal à $400 \mathrm{tr} / \mathrm{min}$.

L'ajout des différents éléments dans le mélange réactionnel est fait successivement. L'eau est introduite en premier, agitée pendant 5 minutes pour stabiliser les conditions opératoires. Ensuite, le produit (cendres volantes ou $\mathrm{CaCO}_{3}$ ) est introduit et agité pendant encore 5 minutes avant l'introduction de l'acide phosphorique. La cinétique de phosphatation est ensuite suivie 
pendant 80 minutes.

Au cours de la réaction, des échantillons de suspension sont prélevés pour analyser le phosphate résiduel en solution après filtration de la suspension à $0,45 \mu \mathrm{m}$. Le phosphate est dosé par la méthode vanadomolybdique ${ }^{[6]}$, une technique colorimétrique en spectrométrie ultraviolet-visible. Le calcium est analysé par absorption atomique. Le produit final, après 80 minutes de réaction, est filtré et séché pour analyse. Les diagrammes de diffraction des rayons $X$ des échantillons ont été enregistrés sur une poudre désorientée à l'aide d'un diffractomètre de type Siemens D5000 (30 mA, 40 $\mathrm{kV}$ ) équipé d'un filtre de nickel, en utilisant la radiation K $\alpha$ du cuivre $(\lambda=I, 543 \AA)$. Le temps d'acquisition des diagrammes dans l'intervalle de $4-84^{\circ}$ en $2 \theta$ a été fixé à trois secondes par pas de $0,02^{\circ}$ (soit 3 h 20 d'acquisition au total). Les phases cristallisées majeures (à partir de quelques \%) des échantillons ont été identifiées par comparaison avec la base de données J.C.P.D.S. Les analyses ponctuelles ont été faites sur une microsonde électronique (MSE) de type Cameca SX 50 pour un potentiel d'accélération égal à $15 \mathrm{kV}$ et un courant de référence de $12 \mathrm{nA}$. Les conditions d'analyse ont été adaptées aux plus faibles teneurs en $\mathrm{Cr}, \mathrm{Pb}$ et $\mathrm{Zn}$. Ainsi, le temps de comptage, classiquement de 6 secondes pour les éléments majeurs, a été porté à 20 secondes pour $\mathrm{Cr}, \mathrm{Zn}$ et $\mathrm{Pb}$.

Les standards utilisés sont :

l'albite pour $\mathrm{NaK} \alpha$ et $\operatorname{SiK} \alpha$,

l'orthose pour $\mathrm{KK} \alpha$,

$\mathrm{Al}_{2} \mathrm{O}_{3}$ pour $\mathrm{AlK} \alpha$,

$\mathrm{MnTiO}_{3}$ pour TiK $\alpha$ et $\mathrm{MnK} \alpha$,

l'andradite pour $\mathrm{CaK} \alpha$,

$\mathrm{Fe}_{2} \mathrm{O}_{3}$ pour $\mathrm{FeK} \alpha$,

la forstérite pour $\mathrm{MgK} \alpha$,

$\mathrm{Cr}_{2} \mathrm{O}_{3}$ pour $\mathrm{CrK} \alpha$,

$\mathrm{FeS}_{2}$ pour SK $\alpha$,

$\mathrm{PbS}$ pour $\mathrm{PbM} \alpha$,

$\mathrm{ZnO}$ pour $\mathrm{ZnK} \alpha$,

fluoroapatite de Durango pour PK $\alpha$ et la vanadinite pour $\operatorname{CIK} \alpha$.

Les corrections ont été faites avec le programme de Pouchou et Pichoir [7].

\section{RÉSULTATS ET DISCUSSION}

\section{$\mathrm{pH}$ et température lors de la réaction de phosphatation}

Lors de l'ajout des cendres volantes dans l'eau, le $\mathrm{pH}$ de la suspension se stabilise vers 10 (figure I). Puis, avec l'ajout de l'acide phosphorique, le $\mathrm{pH}$ chute immédiatement jusqu'à des valeurs voisines de 3 et 4 avant de remonter lentement vers de valeurs comprises entre 5,5 et 6,5. La vitesse de remontée du $\mathrm{pH}$ en début de réaction est fonction de la température initiale du mélange réactionnel (de 20 à $80^{\circ} \mathrm{C}$ ) bien que le $\mathrm{pH}$ en fin d'expérience demeure similaire quelle que soit la température.

Cette évolution du $\mathrm{pH}$ est semblable pour les deux cendres volantes étudiées et elle est proche de celle de la phosphatation de $\mathrm{CaCO}_{3}$ seul (figure I). Cependant, dans le cas du $\mathrm{CaCO}_{3}$, le $\mathrm{pH}$ avant phosphatation se situe vers 8 . Après moins de 3 minutes de phosphatation, le $\mathrm{pH}$ se stabilise à un $\mathrm{pH}$ voisin de 6 . Le temps nécessaire pour atteindre le palier diminue avec l'augmentation de la température de réaction.

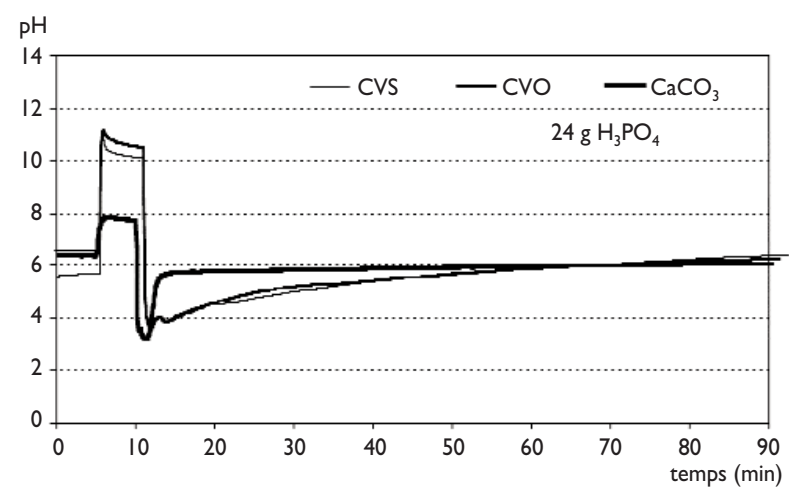

Figure I : Évolution du pH lors de la phosphatation des $\mathrm{CVS}$, des $\mathrm{CVO}$ et du $\mathrm{CaCO}_{3}$ à $20^{\circ} \mathrm{C}$

Un suivi fin de l'évolution de la température dans le réacteur permet de noter :

- une légère augmentation de l'ordre de $1{ }^{\circ} \mathrm{C}$ lors de l'introduction des cendres volantes dans l'eau alors que l'ajout du $\mathrm{CaCO}_{3}$ dans l'eau, n'entraîne aucune modification de température.

- que l'introduction de l'acide phosphorique induit une augmentation importante de la température, de 6 à II ${ }^{\circ} \mathrm{C}$, selon le taux de phosphatation. En effet, l'enthalpie de la réaction de phosphatation des cendres volantes est de $-770 \mathrm{~kJ} / \mathrm{kg}_{\mathrm{H}_{3} \mathrm{PO}}$ pour les CVS et de $-685 \mathrm{~kJ} / \mathrm{kg}_{\mathrm{H}_{3} \mathrm{PO}_{4}}$ pour les $\mathrm{CVO}$. Une faible partie de cette évolution de température (de 1,6 à $3{ }^{\circ} \mathrm{C}$ ) est due à l'exothermicité de la dilution de l'acide phosphorique dans l'eau (enthalpie de $-140 \mathrm{~kJ} / \mathrm{kg}_{\mathrm{H}_{3} \mathrm{PO}_{4}}$ ), l'autre partie étant due à la réaction chimique.

- enfin que la température diminue pour se stabiliser vers la valeur de consigne. L'élévation de température notée lors de la phosphatation est similaire pour les deux cendres volantes et est nettement plus importante que lors de la phosphatation du $\mathrm{CaCO}_{3}, 2,3$ à $4{ }^{\circ} \mathrm{C}$ selon le taux de phosphatation.

\section{Cinétique de phosphatation}

La réaction de phosphatation des cendres volantes est légèrement moins rapide pour les CVS que pour les CVO (figure 2). Globalement, la moitié du phosphate introduit dans la suspension de cendres volantes est consommée pendant la première minute. Puis entre 10 


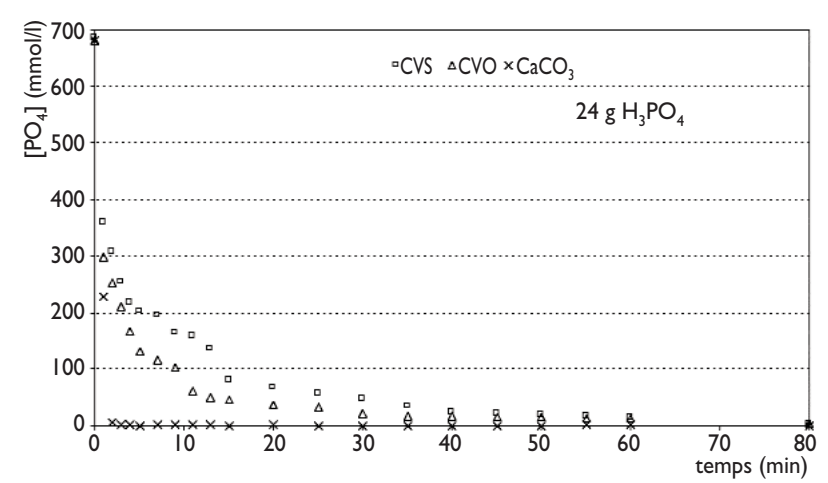

Figure 2 : Cinétique de phosphatation des CVO, des CVS et du $\mathrm{CaCO}_{3}$ à $20^{\circ} \mathrm{C}$

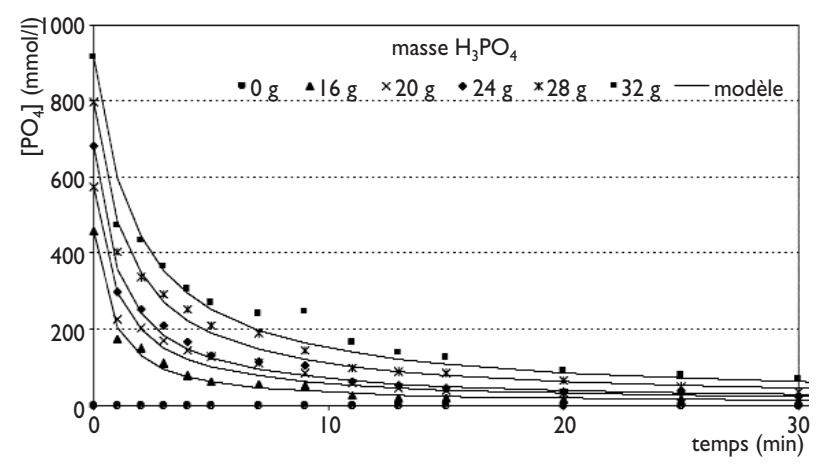

Figure 3 : Cinétique de phosphatation des CVO à taux de phosphatation variable et à $20^{\circ} \mathrm{C}$

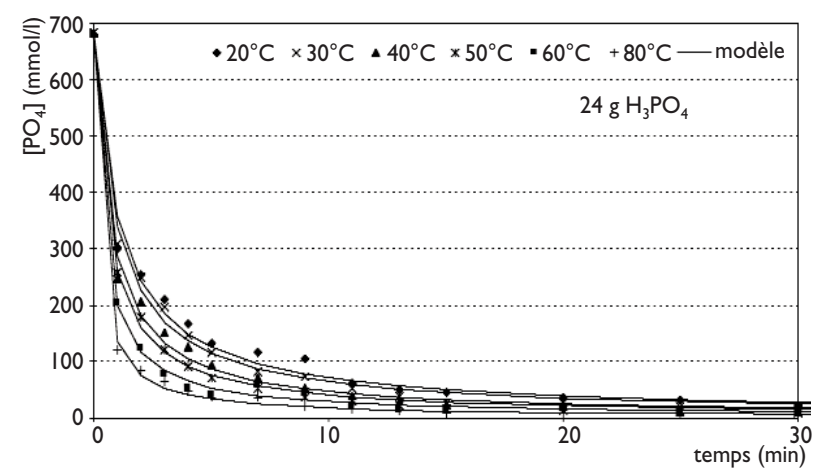

Figure 4 : Cinétique de phosphatation des CVO à températures variables

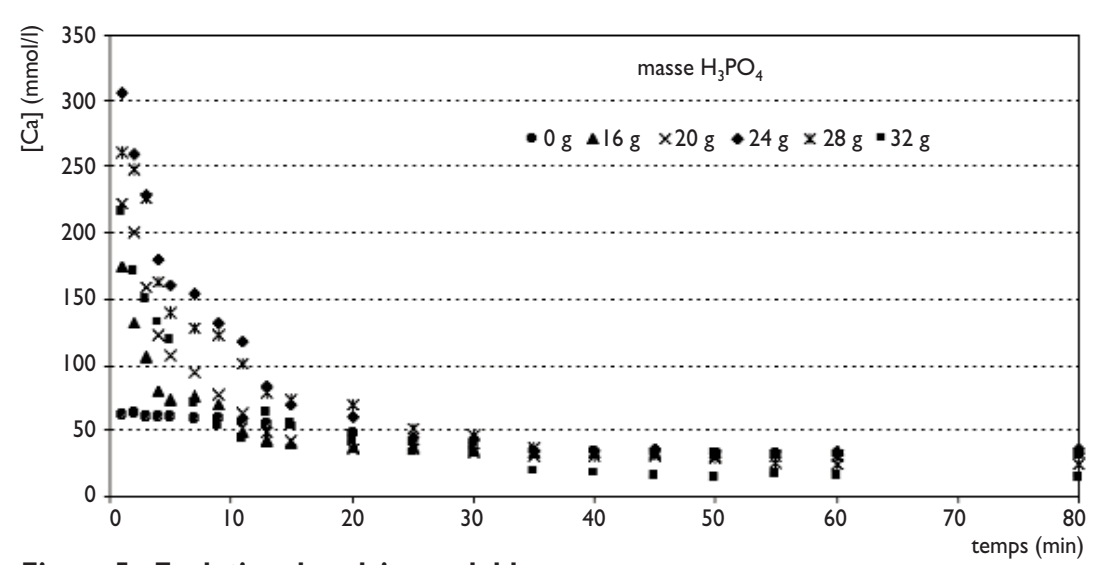

Figure 5 : Evolution du calcium soluble lors de la phosphatation des CVS à $20^{\circ} \mathrm{C}$ et 30 minutes sont nécessaires pour en consommer $40 \%$ de plus. Le phosphate restant est progressivement consommé jusqu'à la fin de l'expérience. Par contre, la phosphatation du $\mathrm{CaCO}_{3}$ est beaucoup plus rapide : l'ensemble du phosphate est consommé en moins de 3 minutes.

En outre, le temps nécessaire pour consommer le phosphate est d'autant plus important que la quantité d'acide phosphorique ajoutée est élevée. Cette observation est illustrée dans la figure 3.

Les résultats présentés sur la figure 4 montrent que l'augmentation de la température de réaction induit une accélération de la réaction de phosphatation.

Lors de la phosphatation des cendres volantes, des quantités importantes de calcium sont présentes en solution dans les premiers instants de la réaction (figure 5). La concentration en calcium diminue ensuite rapidement pour rejoindre la concentration en calcium du mélange cendres et eau non phosphaté. La phosphatation des cendres volantes conduit donc à une dissolution très rapide du calcium initialement contenu dans les cendres. Celui-ci réagit avec le phosphate en solution pour former des phosphates calciques peu solubles. Ce type de réaction peut être décrit par des mécanismes de type dissolution - précipitation dont la vitesse dépend essentiellement de la surface d'échange exprimée sous forme de surface spécifique Sp. Ce mécanisme peut-être représenté par la relation suivante ${ }^{[8,9]}$ :

$$
-\frac{\mathrm{d}\left[\mathrm{PO}_{4}\right]}{\mathrm{dt}}=\text { k.Sp.m. }\left(\left[\mathrm{PO}_{4}\right]-\left[\mathrm{PO}_{4}\right]_{\mathrm{f}}\right)^{2}
$$

Cette équation représente l'évolution de la concentration de phosphate en solution suivant une réaction équilibrée de surface et du second ordre.

La modélisation des données expérimentales montrant la consommation de phosphates par les éléments majeurs des cendres volantes par l'équation (I) permet de déterminer les paramètres cinétiques de la réaction de phosphatation.

La modélisation des données expérimentales effectuée sur Matlab a été comparée à l'expérience. Les résultats présentés sur les figures 3 et 4 montrent une bonne corrélation entre les données expérimentales et modélisées. L'écart observé est inférieur à $25 \%$, ce qui est acceptable compte tenu de l'hétérogénéité constatée pour les cendres volantes.

Les valeurs déterminées pour les constantes de vitesse de réaction $k$ sont comprises entre 0,003 et $0,035 \mathrm{l} / \mathrm{mol} . \mathrm{s} . \mathrm{m}^{2}$. Cette constante diminue légèrement avec l'augmen- 
tation du taux de phosphatation et elle augmente avec la température, ce qui est conforme à la loi d'Arrhénius qui montre une accélération de la cinétique de réaction avec l'augmentation de la température. L'évolution de la constante de vitesse de réaction avec la température (de 20 à $80^{\circ} \mathrm{C}$ ) permet de définir, par cette loi, l'énergie d'activation de la réaction de phosphatation des cendres volantes. L'énergie d'activation est de $19,7 \mathrm{~kJ} / \mathrm{mol}$ pour les cendres CVS et de $21,8 \mathrm{~kJ} / \mathrm{mol}$ pour les cendres CVO. Les énergies d'activation sont du même ordre de grandeur que celle obtenue pour la phosphatation du $\mathrm{CaCO}_{3}(15,8 \mathrm{~kJ} / \mathrm{mol})$. La phosphatation du $\mathrm{CaCO}_{3}$ a été modélisée par une loi similaire aux cendres volantes mais d'ordre I. Cette différence est attribuée aux mécanismes plus complexes qui ont lieu lors de la phosphatation des cendres volantes comme le démontre les différences constatées lors des comparaisons entre $\mathrm{CV}$ et carbonate de calcium (figure I).

Ainsi, en dépit d'une différence de composition importante, en ce qui concerne les sulfates et les métaux lourds, les deux cendres étudiées se comportent de la façon similaire : les valeurs de constantes de vitesse de réaction sont très proches. Les surfaces spécifiques voisines laissent penser que la phosphatation est surtout contrôlée par une réaction en surface. En effet, les faibles énergies d'activation déterminées (environ $20 \mathrm{~kJ} / \mathrm{mol}$ ) impliquent que la phosphatation des cendres volantes correspond à une réaction chimique de neutralisation acido-basique. Le fait de prendre en compte la surface de contact entre les particules et la solution permet de définir une loi globale de la cinétique de réaction de phosphatation des cendres volantes pouvant rendre compte du comportement de plusieurs types de cendres volantes. La vitesse de consommation du phosphate en solution lors de la phosphatation des CVO est plus rapide que celle des CVS du fait de la plus grande surface spécifique des CVO.

\section{ÉVALUATION DU PROCÉDÉ}

Le but de la phosphatation des cendres volantes est d'insolubiliser les métaux lourds par la formation de complexes phosphatés stables.

Les analyses en diffraction des rayons $X(D R X)$ montrent qu'aucune nouvelle phase cristalline n'est présente lors de la phosphatation des cendres volantes. En effet, celles-ci sont toujours principalement constituées d'anhydrite, de gehlenite et de carbonate de calcium avec du quartz, des plagioclases et des phosphates peu cristallins également présents en plus faible quantité. La présence de phases amorphes, à l'origine dans les cendres volantes, ne permet pas de mettre en évidence une variation de la quantité de phospha- te non cristallin avec le taux de phosphatation. Une légère diminution de l'intensité du pic de carbonate de calcium peut toutefois être constatée lors de la phosphatation des cendres volantes.

Par contre l'analyse en $\mathrm{DRX}$ du $\mathrm{CaCO}_{3}$ phosphaté montre que la quantité de $\mathrm{CaCO}_{3}$ diminue avec l'augmentation du taux de phosphatation (figure 6). Puis, lorsqu'il est phosphaté, un pic mal défini correspondant à des phosphates calciques de type apatitique apparaît ainsi que du phosphate octocalcique $\left(\mathrm{Ca}_{8} \mathrm{H}_{2}\left(\mathrm{PO}_{4}\right)_{6}\right.$, $5 \mathrm{H}_{2} \mathrm{O}$ ). La réaction de phosphatation du $\mathrm{CaCO}_{3}$ a donc permis la formation de phosphate calcique suivant les réactions suivantes [5] :

$$
\begin{aligned}
& \mathrm{CaCO}_{3}+ \mathrm{H}_{3} \mathrm{PO}_{4}+\mathrm{H}_{2} \mathrm{O} \leftrightarrow \mathrm{CaHPO}_{4}, 2 \mathrm{H}_{2} \mathrm{O}+\mathrm{CO}_{2} \\
& 6 \mathrm{CaHPO}_{4}, 2 \mathrm{H}_{2} \mathrm{O}+2 \mathrm{CaCO}_{3} \\
& \leftrightarrow \mathrm{Ca}_{8} \mathrm{H}_{2}\left(\mathrm{PO}_{4}\right)_{6}, 5 \mathrm{H}_{2} \mathrm{O}+2 \mathrm{CO}_{2}+9 \mathrm{H}_{2} \mathrm{O} \\
& \mathrm{Ca}_{8} \mathrm{H}_{2}\left(\mathrm{PO}_{4}\right)_{6}, 5 \mathrm{H}_{2} \mathrm{O}+2 \mathrm{CaCO}_{3} \\
& \leftrightarrow \mathrm{Ca}_{10}\left(\mathrm{PO}_{4}\right)_{6}(\mathrm{OH})_{2}+2 \mathrm{CO}_{2}+5 \mathrm{H}_{2} \mathrm{O}
\end{aligned}
$$

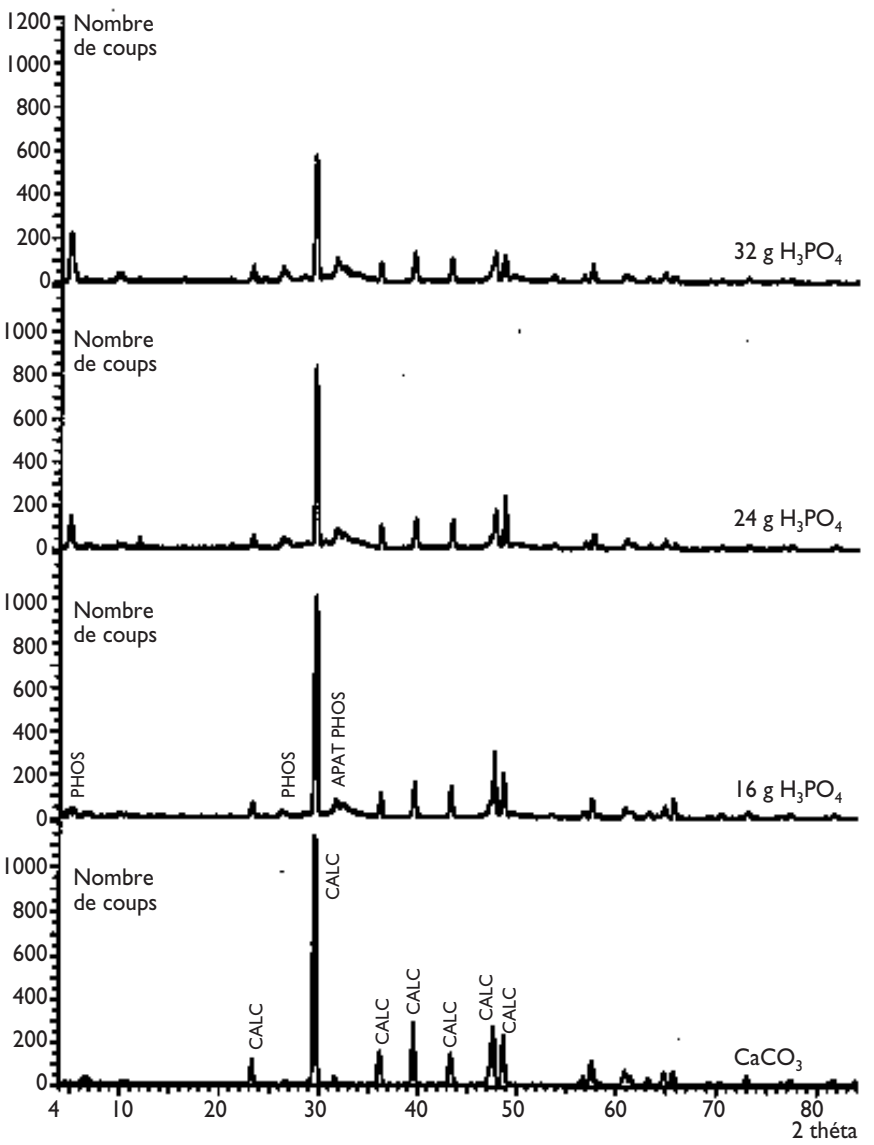

Figure 6 : Diffractogrammes des rayons $X$ des produits de phosphatation du $\mathrm{CaCO}_{3}$

CALC : calcite $\left(\mathrm{CaCO}_{3}\right)$

PHOS : phosphate octocalcique $\left(\mathrm{Ca}_{8} \mathrm{H}_{2}\left(\mathrm{PO}_{4}\right)_{6}, 5 \mathrm{H}_{2} \mathrm{O}\right)$

APAT : phosphate calcique amorphe apatitique 
L'analyse en microsonde des cendres phosphatées montre que le phosphate disparu de la phase liquide a bien réagi avec les particules ou est adsorbé sur leur surface. La phosphatation ne modifie pas la répartition des éléments majeurs des cendres ( $\mathrm{Si}, \mathrm{Ca}$ et $\mathrm{Al}$ ). Les silicoaluminates, la silice, l'aluminium et les sulfates de calcium ont plutôt tendance à être enrobés en surface par du phosphate. Le phosphate est principalement associé au calcium.

Certaines particules de cendres volantes initiales ou phosphatées ont été étudiées en microanalyse quantitative ponctuelle (quelques $\mu \mathrm{m}^{3}$ ) à la MSE. A partir de ces analyses, des associations minéralogiques ont été déterminées suivant des tests logiques basés sur la stœchiométrie des minéraux. Cette technique de calcul a déjà été utilisée pour la caractérisation de résidus d'incinération [10]. Les associations minéralogiques déterminées sont les carbonates, les feldspaths, les mélilites, les perowskites, les phosphates, la silice, les sulfates et les mélanges de titane et de zinc. Ces minéraux correspondent à ceux déterminés dans d'autres cendres volantes [10]. Les phosphates et les sulfates correspondent souvent à des mélanges "mécaniques" de minéraux car la taille du faisceau électronique incident fait que plusieurs minéraux distincts peuvent être excités et donc analysés simultanément.

La représentation triangulaire de l'ensemble des données permet de visualiser l'évolution de la composition des particules lors de la phosphatation. Ces données sont représentées par leur association minéralogique précédemment définie. La position des points représente la proportion relative des 3 éléments purs $(\mathrm{CaO}$, $\mathrm{P}_{2} \mathrm{O}_{5}$ ou $\mathrm{SiO}_{2}$ ), situés à chaque pôle du triangle, contenu dans chaque analyse. Typiquement les sulfates de calcium $\left(\mathrm{CaSO}_{4}\right)$ contiennent du $\mathrm{CaO}$, mais pas de $\mathrm{P}_{2} \mathrm{O}_{5}$ ni de $\mathrm{SiO}_{2}$, ils sont donc localisés vers le pôle $\mathrm{CaO}$. Les analyses des cendres initiales montrent, dans un diagramme $\mathrm{CaO}$ $\mathrm{P}_{2} \mathrm{O}_{5}-\mathrm{SiO}_{2}$, une composition principalement située entre les pôles $\mathrm{CaO}$ et $\mathrm{SiO}_{2}$ (figure 7). Par contre, après la phosphatation, la majorité des analyses se localise dans la moitié du triangle de la médiane issue du pôle $\mathrm{SiO}_{2}$ vers le pôle $\mathrm{CaO}$ (figure 8$)$. La répartition des phosphates est assez homogène dans cette partie du triangle. Le traitement par phosphatation induit la formation de nombreux phosphates de rapport $\mathrm{CaO} / \mathrm{P}_{2} \mathrm{O}_{5}$ variable. $\mathrm{Ce}$ rapport $\mathrm{CaO} / \mathrm{P}_{2} \mathrm{O}_{5}$ caractérise les différentes espèces phosphocalciques. Pour les deux cendres étudiées, une grande partie des phosphates formés a un rapport $\mathrm{CaO} / \mathrm{P}_{2} \mathrm{O}_{5}$ compris entre 0,8 et $\mathrm{I}, 3$ : il s'agit de phosphates de type dicalcique $\left(\mathrm{CaHPO}_{4}\right)$ à apatitique (figure 9). Cependant, certaines compositions de phosphates présentent des rapports $\mathrm{CaO} / \mathrm{P}_{2} \mathrm{O}_{5}$ nettement plus élevés à cause des mélanges "mécaniques" signalés plus haut dans le texte. La phosphatation des cendres volantes conduit à la formation d'une matrice phosphatée complexe dont certains éléments forment des phosphates qui pourront évoluer naturellement vers les phases de type apatitique, les moins solubles et donc les plus stables.

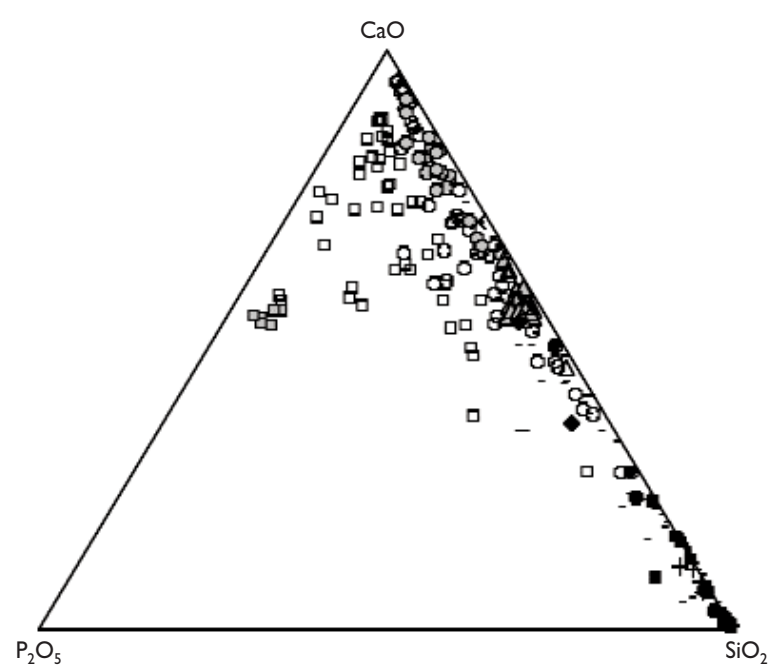

- autres

$\diamond$ Carbonate

- Chlorure

+ Feldspath

$\Delta$ Mélilite

- Oxyde d'Al

$\times$ Perowskite

口 Phosphate

口 Phosphate M

- Pyroxène

- Sulfate $M$

- Sulfate

$\Delta$ Titane $M$

- Zinc M

Figure 7 : Diagrammes triangulaires $\mathrm{CaO}-\mathrm{P}_{2} \mathrm{O}_{5}-\mathrm{SiO}_{2}$ des CVO initiales ( $M$ : mélange)

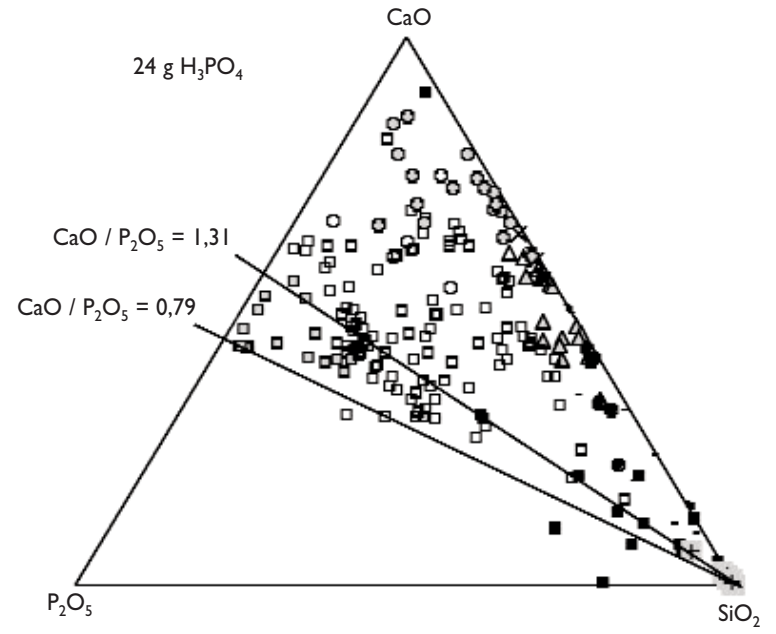

- autres

- Chlorure

$\Delta$ Mélilite

- Oxyde d'Al

$\times$ Perowskite

口 Phosphate

a Phosphate M

- Pyroxène

+ Silice

S Sulfate $M$

- Sulfate

$\Delta$ Titane $M$

Figure 8 : Diagrammes triangulaires $\mathrm{CaO}-\mathrm{P}_{2} \mathrm{O}_{5}-\mathrm{SiO}_{2}$ des CVO phosphatées ( $M$ : mélange) 
La répartition des espèces formées, illustrée dans la figure 9, montre en effet que le système n'a pas encore atteint un équilibre thermodynamique optimal représenté par le phosphate de nature apatitique pour les conditions expérimentales évoquées. II est très probable que la calcination qui normalement suit cette phase du procédé améliorera grandement la stabilité du système phosphate [5].

\section{CONCLUSIONS}

Pour mieux comprendre le traitement chimique des cendres volantes d'incinération d'ordures ménagères par de l'acide phosphorique, la cinétique de phosphatation a été étudiée à partir de deux cendres volantes différentes et du carbonate de calcium. La phosphatation de ces produits correspond à une réaction de dissolution-précipitation qui consomme rapidement l'ensemble du phosphate introduit au cours de l'expérience. La phosphatation du carbonate de calcium ressemble en de nombreux points à la réaction de phosphatation des cendres volantes. Les résultats obtenus montrent que ce composé modèle, qui est aussi présent dans les cendres volantes, joue un rôle prépondérant lors de la phosphatation des cendres volantes, bien que le $\mathrm{CaCO}_{3}$ ne soit pas le seul élément à réagir lors de ce traitement.

La cinétique de phosphatation des cendres volantes se modélise correctement par une réaction réversible d'ordre 2. Les faibles énergies d'activation de cette réaction, environ $20 \mathrm{~kJ} / \mathrm{mol}$, sont caractéristiques des processus physiques et de neutralisation acido-basique ne nécessitant pas une énergie importante.

La phosphatation des cendres volantes induit la formation de phosphates calciques qui enrobent les particules moins réactives. Ces phosphates calciques contribuent à la stabilisation des métaux lourds contenus dans les cendres volantes.

\section{Remerciements}

Ce travail a pu être réalisé grâce au soutien financier du département Health, Safety, Environment - Direction centrale recherche et technologie du groupe Solvay.

Blandine Bournonville, doctorante,

Ange Nzihou, enseignant chercheur,

Laboratoire de génie des procédés des solides divisés UMR CNRS 2392, École des mines d'Albi-Carmaux, Campus Jarlard, Route de Teillet, 81013 Albi CT cedex 09, France

Patrick Sharrock, professeur

IUT Castres - Université Paul Sabatier, Avenue Georges Pompidou BP 258, 81104 Castres Cedex, France

\section{Guy Depelsenaire,}

HSE (health, safety, environment), Direction centrale recherche et technologie, Solvay, Rue de Ransbeek 310, B-I I 20 Bruxelles, Belgique

\section{Nomenclature}

$\mathrm{k} \quad$ constante de vitesse de réaction $\left(\mathrm{I} / \mathrm{mol} . \mathrm{s} . \mathrm{m}^{2}\right)$

m masse des particules (g)

$\left[\mathrm{PO}_{4}\right]$ concentration en phosphate en solution $(\mathrm{mol} / \mathrm{l})$

$\left[\mathrm{PO}_{4}\right]_{\mathrm{f}}$ concentration finale en phosphate en solution $(\mathrm{mol} / \mathrm{l})$

$\mathrm{Sp} \quad$ surface spécifique des particules $\left(\mathrm{m}^{2} / \mathrm{g}\right)$

$\mathrm{t}$ temps (s)

\section{Bibliographie}

[I] R. Derie, A new way to stabilize fly ash from municipal incinerators. Waste Management, 16, 8, 7II-716, 1996.

[2] R. Derie et C. Depaus. Sur le comportement des polluants minéraux et organique au cours du Procédé Neutrec d'inertage des cendres volantes d'incinérateurs d'ordures ménagères, Proceeding de Stab \& Env 99, 269-273, 1999.

[3] T.T. Eighmy, B.S. Crannell, J.E. Krzanowski, L.G. Butler, F.K. Cartledge, E.F. Emery, J.D. Eudsen Jr, E.L. Shaw and C.A. Francis, Characterization and phosphate stabilization of dusts from the vitrification of MSW combustion residues. Waste Management, I8, 5|3-524, 1998.

[4] A. Nzihou and P. Sharrock, Calcium phosphate stabilization of fly ash with choride extraction. Waste Management, 22, 235-239, 2002.

[5] P. Piantone, F. Bodénan, R. Derie and G. Depelsenaire, Monitoring the stabilization of municipal solid waste incineration fly ash by phosphatation : mineralogical and balance approach. Waste Management, 23 (3), 225-243, 2003.

[6] B. Bournonville, Stabilisation des métaux lourds dans les cendres volantes d'incinération - comportement rhéologique, cinétique de phosphatation et évaluation du procédé. Thèse de doctorat, Université de Perpignan, 19 décembre 2002.

[7] ].L. Pouchou et F. Pichoir, Un nouveau modèle de calcul pour la microanalyse quantitative par spectrométrie de RX. Partie I. Application à l'analyse des échantillons homogènes. La recherche aérospatiale, 6 (3), 167-192, 1984.

[8] N.S. Tavare. Batch Crystallizers, Reviews in Chemical Engineering, Freund Publishing House Ltd, London, England, 7, 3-4, 199I.

[9] J. Garside, A. Mersmann, J. Nyvlt, Measurement of crystal growth rates, European Federation of Chemical Engineering Working Party on Crystallization, 1990. 